\title{
Caring for cancer survivors: perspectives of oncologists, general practitioners and patients in Italy
}

\author{
Fabio Puglisi ${ }^{\ddagger 1,2}$, Elisa Agostinetto ${ }^{1,2}$, Lorenzo Gerratana ${ }^{* 1,2}$, Claudia Bozza, ${ }^{1,2}$, \\ Maurizio Cancian ${ }^{3}$, Elisabetta lannelli ${ }^{4}$, Giovanni Ratti ${ }^{5}$, Saverio Cinieri ${ }^{\S, 6,7}$ \\ \& Gianmauro Numico ${ }^{\ddagger}$
}

\begin{abstract}
Aim: The present survey investigates the views of medical oncologists, general practitioners (GPs) and patients about the various surveillance strategies. Methods: An online survey was conducted in Italy on a population of 329 medical oncologists, 380 GPs and 350 patients. Results: Most of GPs ( $\mathrm{n}=291 ; 76 \%)$ claim that follow-up should be provided by the collaboration between GPs and medical oncologists. Most medical oncologists report to have a poor relationship with GPs $(n=151 ; 46 \%)$ or no relationships at all ( $n=14 ; 4 \%)$. Most patients believe there is no real collaboration between medical oncologists and GPs $(n=138 ; 54 \%)$. Conclusion: GPs, medical oncologists and patients share the idea that the collaboration between oncologists and GPs for surveillance of cancer survivors is poor and should be improved.
\end{abstract}

First draft submitted: 15 August 2016; Accepted for publication: 1 September 2016; Published online: 12 September 2016

The increasing number of individuals who received a cancer diagnosis and the improvement in survival rates are leading to a growing number of cancer survivors. In Italy, in 2015, 363,000 new cases were diagnosed, almost 1000 each day [1]. On the other hand, the diagnostic and therapeutic advances lead to a decrease in mortality rate that dropped as much as 20\% from 1996. This requires maximizing efficacy and efficiency of caring for people living with, and beyond, cancer [2-4]. According to the National Coalition for Cancer Survivorship, a 'cancer survivor' is defined as anyone with a history of cancer, from the time of diagnosis and for the remainder of life, whether that is days or decades. It is estimated that in the USA in 2022 the population of cancer survivors will increase to nearly 18 million [5]. In Italy, the estimated number of cancer survivors in 2015 was 3 million [1]. The Cochrane Breast Cancer Group defines 'follow-up' as the regular use of laboratory or instrumental tests in otherwise asymptomatic patients to detect distant metastases earlier $[6,7]$. This interpretation does not include other activities, such as the evaluation of long-term treatment side effects, psychological interventions and lifestyle corrections, that are part of a more comprehensive approach which is usually referred as 'survivorship care' [8,9].

'Department of Medical \& Biological Sciences, University of Udine, Udine, Italy

${ }^{2}$ Department of Medical Oncology, University Hospital of Udine, Udine, Italy

${ }^{3}$ Società Italiana di Medicina Generale e delle Cure Primarie (SIMG) - Italian College of General Practitioner, Conegliano, TV, Italy

${ }^{4}$ Italian Federation of Volunteer-based Cancer Organizations FAVO, Rome, Italy

${ }_{5}^{5}$ Segreteria Associazione Italiana di Oncologia Medica (AIOM)/AIOM Secretariat Staff, Milan, Italy

${ }^{6}$ Medical Oncology Division \& Breast Unit, Brindisi, Italy

${ }^{7}$ Medicine Department IEO (Istituto Europeo di Oncologia) IRCCS, Milan, Italy

${ }^{8}$ Medical Oncology Unit, SS Antonio \& Biagio \& C Arrigo Hospital, Alessandria, Italy

*Author for correspondence: Tel.: + 39432 552754; Fax: +39 432 552762; gerratana.lorenzo@spes.uniud.it

${ }^{\ddagger}$ Member of Associazione Italiana di Oncologia Medica (AIOM), Italy

${ }^{5}$ National Treasurer of Associazione Italiana di Oncologia Medica (AIOM), Italy

\section{KEYWORDS}

- cancer survivors

- follow-up • general practitioners $\bullet$ oncologists

- patients • surveillance 
Unfortunately, a gold standard in surveillance management is currently lacking and it is even unclear whether the oncologist or the general practitioner (GP) should provide the follow-up care $[4,10-11]$. A sequential approach has been largely suggested and consists in a specialist phase, usually carried out by the oncologist, followed by a local phase, managed by the GP [12] Nevertheless, this approach is not universally adopted, and there are some risks due to poor communication among different physicians that may lead to poor quality of surveillance with underuse of appropriate tests and visits or a duplication of care and abuse of resources [13,14]. The overuse of imaging and laboratory testing may cause anxiety to the patient, because of the risk of false-positive results, and translates into higher costs [15,16]. This approach is often led by the unrealistic belief that more testing might anticipate diagnosis of recurrence, giving a false feeling of reassurance to the patient $[17,18]$.

A Canadian study showed that on a population of breast cancer survivors, 5 years after primary treatment, the mean number of visits was higher than recommended by the guidelines. For example, during the second year patients underwent a mean of 11.2 visits compared with two to four visits recommended. These visits were performed by different physicians such as the medical oncologist, the surgeon and the GP [16]. A questionnaire sent to 562 specialists registered in the Cancer Research Clinical Trials Unit database pointed out that specialists tend to adopt a risk adjusted surveillance strategy. Moreover, the study showed that lack of GPs' cancer expertise concerns significantly medical oncologists [11].

A Canadian randomized study conducted in 2006 on a population of 968 early breast cancer women showed that follow-up could be managed by the GP with no negative relapses on overall survival or quality of life [12]. Patient's preferences should be considered as a key point on the organization of follow-up strategy. Bell et al.'s study analyzes the reasons which lead a patient to prefer either the medical oncologist or the GP. Concerns expressed by US cancer survivor about follow-up care from a primary care physician include: lack of cancer expertise, that the GP was not involved in their initial treatment and that there may be lack of continuity of care [19]. Brennan et al. reported a closer involvement of the GP resulted in an improvement of patients' logistics and doctor-patient relationship [20]. These examples provide the message that patients' wishes should be taken into consideration and support the idea that a personalized approach could be considered.

There is little evidence about patients' perception about follow-up care and little is known about the point of view of both medical oncologists and GPs [19-22]. There are studies showing that a large percentage of oncologists do not believe GPs have the skills to conduct appropriate testing for cancer recurrence [23,24]. Also patients often have an unfavorable view regarding a central role for GPs in cancer follow-up [25]. Some GPs are willing to assume responsibility in cancer follow-up and their willingness seems to vary according to prior involvement with cancer survivorship [26,27]. Opinions may be related to the specific healthcare system of a country. Italian data about this topic are currently lacking. Aim of the study is to describe patients', medical oncologists' and GPs' perspectives on the follow-up management in the Italian setting. Furthermore, it focuses on factors that could influence medical oncologists' answers.

\section{Methods}

A questionnaire has been available online for 45 days (from the 16 July to 1 September 2014) and questions were differentiated for each subset of respondents. Perceptions of follow-up, opinions on surveillance strategy and quality of relationship with colleagues were investigated. Questionnaires could be filled online or sent by e-mail. The oncologists' survey was sent to the 2210 members of the Italian Association of Medical Oncology (AIOM). Patients were involved thanks to the Italian Federation of Voluntary Work Associations in Oncology and the Italian Association of Cancer Patients through online media and informational desks in the Hospitals and in the Research Institutes involved in the study. A total of 2736 GPs were invited by mail to fill the online questionnaire by the Italian College of General Practitioner (SIMG).

The demographic data of the survey sample were summarized through descriptive analysis (Table 1). All the questions and answers provided are reported in Table 2 .

We further analyzed the oncologists' subset in order to explore the association between specific characteristics and answers given in the 
Table 1. Baseline characteristics by group.

\section{Characteristics}

General practitioners $(n=380)$

\section{Gender:}

- Male

- Female

Age:

- More than 55 years

- Between 40 and 55 years

- Less than 40 years

Years of profession:

- More than 20 years

- Between 10 and 20 years

- Between 5 and 10 years

- Less than 5 years

Number of patients:

- More than 1000

- Between 500 and 1000

- Less than 500

Region of activity:

- North

- Center

- South and Islands

City of activity:

- Less than 15,000 inhabitants

- Between 15,000 and 50,000

- More than 50,000 inhabitants

Distance from cancer center:

- Less than $10 \mathrm{~km}$

- Less than $30 \mathrm{~km}$

- Less than $50 \mathrm{~km}$

Daily activity:

- Alone

- With a dedicated staff

- With others GPs

- With a dedicated staff and other GPs

Medical oncologists $(n=329)$

Gender:

- Male

- Female

Age:

- Younger than 40 years old

- Between 40 and 55 years old

- Older than 55 years old

Years of profession:

- Less than 5 years

- Between 5 and 10 years

- Between 10 and 20 years

- More than 20 years

GM: General practitioner. n $\%$

$70 \%$

$30 \%$

114

$77 \%$

$\begin{array}{ll}294 & 77 \% \\ 82 & 22 \%\end{array}$

$4 \quad 1 \%$

$305 \quad 80 \%$

$60 \quad 16 \%$

$7 \quad 2 \%$

$8 \quad 2 \%$

$337 \quad 89 \%$

$36 \quad 9 \%$

$7 \quad 2 \%$

$247 \quad 65 \%$

$49 \quad 13 \%$

$84 \quad 22 \%$

$151 \quad 40 \%$

$91 \quad 24 \%$

$138 \quad 36 \%$

$246 \quad 65 \%$

$104 \quad 27 \%$

$30 \quad 8 \%$

$154 \quad 40 \%$

$70 \quad 18 \%$

$41 \quad 11 \%$

$185 \quad 49 \%$

$163 \quad 50 \%$

$166 \quad 50 \%$

$113 \quad 34 \%$

$120 \quad 36 \%$

$96 \quad 30 \%$

$77 \quad 23 \%$

$36 \quad 11 \%$

$79 \quad 24 \%$

$125 \quad 38 \%$ 
RESEARCH ARTICLE Puglisi, Agostinetto, Gerratana et al.

\begin{tabular}{|c|c|c|}
\hline Characteristics & n & $\%$ \\
\hline \multicolumn{3}{|c|}{ Medical oncologists ( $n=329$ ) (cont.) } \\
\hline \multicolumn{3}{|l|}{ Institution: } \\
\hline - General hospital & 243 & $73 \%$ \\
\hline - Research institute & 48 & $15 \%$ \\
\hline - Private clinic & 3 & $1 \%$ \\
\hline - Other & 35 & $11 \%$ \\
\hline \multicolumn{3}{|l|}{ Area of activity: } \\
\hline - North & 167 & $51 \%$ \\
\hline - Center & 76 & $23 \%$ \\
\hline - South and Islands & 86 & $26 \%$ \\
\hline \multicolumn{3}{|l|}{ Professional position: } \\
\hline - Medical manager & 69 & $21 \%$ \\
\hline - Full-time consultant & 175 & $53 \%$ \\
\hline - Fellow & 41 & $12 \%$ \\
\hline - Resident & 44 & $14 \%$ \\
\hline \multicolumn{3}{|l|}{ Area of interest: } \\
\hline - Medical oncology & 316 & $96 \%$ \\
\hline - Radiation oncology & 1 & $0 \%$ \\
\hline - Surgery & 2 & $1 \%$ \\
\hline - Pneumology & 2 & $1 \%$ \\
\hline - Internal medicine & 1 & $0 \%$ \\
\hline - Other & 8 & $2 \%$ \\
\hline \multicolumn{3}{|l|}{ Patients $(n=350)$} \\
\hline \multicolumn{3}{|l|}{ Gender: } \\
\hline - Male & 224 & $64 \%$ \\
\hline - Female & 126 & $36 \%$ \\
\hline \multicolumn{3}{|l|}{ Age: } \\
\hline - Younger than 40 years & 40 & $11 \%$ \\
\hline - Between 40 and 55 years & 154 & $44 \%$ \\
\hline - Older than 55 years & 156 & $44 \%$ \\
\hline \multicolumn{3}{|l|}{ Years from diagnosis: } \\
\hline - Less than 2 years & 106 & $30 \%$ \\
\hline - Between 2 and 5 years & 110 & $31 \%$ \\
\hline - Between 5 and 10 years & 68 & $19 \%$ \\
\hline - More than 10 years & 58 & $17 \%$ \\
\hline \multicolumn{3}{|l|}{ Cancer therapy in progress: } \\
\hline - Yes & 122 & $35 \%$ \\
\hline- No & 226 & $64 \%$ \\
\hline \multicolumn{3}{|c|}{ Time from beginning of follow-up: } \\
\hline-6 months & 88 & $25 \%$ \\
\hline-1 year & 36 & $10 \%$ \\
\hline-2 years & 88 & $25 \%$ \\
\hline-5 years & 40 & $12 \%$ \\
\hline - More than 5 years & 98 & $28 \%$ \\
\hline
\end{tabular}

survey. Chi square test or Fisher's exact test was used as appropriate. Significant associations ( $\mathrm{p} \leq 0.05)$ between social-demographic features and answers provided by medical oncologists are shown in Table 3.

\section{Results}

The national survey was conducted on a population of 1059 participants: 329 medical oncologists (31\%), 380 GPs (36\%) and 350 patients (33\%). 
Table 2. General practitioners', medical oncologists' and patients' perspectives.

\section{GPs'questionnaire}

How many cancer patients do you have?

- Less than 30

- Between 30 and 60

- More than 60

Which physician takes charge of their follow-up?

- A collaboration between medical oncologists and GPs 286 (75)

- Medical oncologist 24 (6)

- GP 13 (3)

- Different physicians (medical oncologists, surgeons, radiation oncologists...) 57 (16)

What's your opinion on follow-up investigation?

- Patients undergo an excessive number of laboratory and instrumental tests 339 (89)

$\begin{array}{ll}- \text { Testing is adequate } & 25 \text { (7) }\end{array}$

- Testing is insufficient

$16(4)$

Provide a percentage to each statement (total sum $=100 \%$ )

The patient feels to be reassured by laboratory tests and imaging:

- From 0 to $25 \%$

- From 26 to $50 \%$

- From 76 to $100 \%$

The patient is worried and has anxiety due to clinical visits and tests:

- From 0 to $25 \%$

- From 26 to $50 \%$

- From 51 to $75 \%$

- From 76 to $100 \%$

The patient, when close to diagnosis, is reassured from tests, after he becomes worried

- From 0 to $25 \%$

- From 26 to $50 \%$

- From 51 to $75 \%$

- From 76 to $100 \%$

After 2-3 years of disease-free survival, who should take charge of patients' follow-up?

- GPs and medical oncologists in collaboration and it should be personalized

- Medical oncologist

- Others

What is your opinion on collaboration with medical oncologists?

- It is very important

- It is quite important

- It is of little importance

At the fourth point of GPS' questionnaire, GPs were asked to provide an agree percentage to each of three statement (statement 1: the patient feels to be reassured by laboratory tests and imaging; statement 2: the patient is worried and had anxiety due to clinical visits and tests; statement 3: the patient, when close to diagnosis, is reassured from tests, after he becomes worried). The total sum had to be $100 \%$. The second point of medical oncologists' questionnaire aimed to figure out the best role of GPs in cancer patient care. Medical oncologists had to indicate a priority order in some tasks provided (promotion of prevention, risk

individuation, follow-up management, therapies' side effects management, palliative care).

The table shows the percentage of oncologists providing a certain priority level for each task (from most important - number 1 - to least important - number 5). The fifth point of medical oncologists' questionnaire is related to GPs as follow-up providers. Medical oncologists were asked to order by relevance some factors that influence the possibility for a GP to become the main follow-up provider. These factors were: cancer type, cancer therapies in progress, patient's wish, year of diagnosis. The table shows the percentage of oncologists providing a certain level of relevance for each factor.

AIOM: Italian Association of Medical Oncology; GP: General practitioner; SIMG: Italian College of General Practitioner. 
Table 2. General practitioners', medical oncologists' and patients' perspectives (cont.).

Items

n (\%)

GPs' questionnaire (cont.)

How much help could be provided by administration offices and a dedicated staff in GPs'

follow-up management?

$\begin{array}{ll}\text { - Very much } & 181(48) \\ \text { - Enough } & 136(36) \\ \text { - A little } & 51(13) \\ \text { - Not at all } & 12(3)\end{array}$

Medical oncologists' questionnaire

How is your relationship with GPs?

- Absent

- Poor

- Good

$141(43)$

- Excellent

$23(7)$

Which is the best role of GP in cancer patients' care? Indicate a priority order in the following activities:

Promotion of prevention:

- Priority level 1

- Priority level 2

- Priority level 3

- Priority level 4

- Priority level 5

- Did not answer

Risk individuation:

- Priority level 1

- Priority level 2

- Priority level 3

- Priority level 4

- Priority level 5

- Did not answer $20(6)$

Follow-up management:

- Priority level 1

- Priority level 2

- Priority level 3

- Priority level 4

- Priority level 5

40 (12)

- Did not answer $21(6)$

Therapies' side effects management

- Priority level 1

- Priority level 2

- Priority level 3

- Priority level 4

- Priority level 5

At the fourth point of GPs' questionnaire, GPs were asked to provide an agree percentage to each of three statement (statement 1: the patient feels to be reassured by laboratory tests and imaging; statement 2: the patient is worried and had anxiety due to clinical visits and tests; statement 3: the patient, when close to diagnosis, is reassured from tests, after he becomes worried). The total sum had to be $100 \%$. The second point of medical oncologists' questionnaire aimed to figure out the best role of GPs in cancer patient care. Medical oncologists had to indicate a priority order in some tasks provided (promotion of prevention, risk individuation, follow-up management, therapies' side effects management, palliative care).

The table shows the percentage of oncologists providing a certain priority level for each task (from most important - number 1 - to least important - number 5). The fifth point of medical oncologists' questionnaire is related to GPs as follow-up providers. Medical oncologists were asked to order by relevance some factors that influence the possibility for a GP to become the main follow-up provider. These factors were: cancer type, cancer therapies in progress, patient's wish, year of diagnosis. The table shows the percentage of oncologists providing a certain level of relevance for each factor.

AIOM: Italian Association of Medical Oncology; GP: General practitioner; SIMG: Italian College of General Practitioner 
Table 2. General practitioners', medical oncologists' and patients' perspectives (cont.).

Medical oncologists' questionnaire (cont.)

Therapies' side effects management (cont.):

- Did not answer

Palliative care:

- Priority level 1

- Priority level 2

$46(14)$

- Priority level 3

$86(26)$

- Priority level 4

$94(29)$

- Priority level 5

$56(17)$

- Did not answer

$15(4)$

Do you think there is a proper integration between hospital and local care?

- Yes

$130(40)$

- No

$187(57)$

- Do not know

$12(3)$

After how long GPs may replace medical oncologists in follow-up management?

-3 years

-5 years

-10 years

- Never

- It depends on the patient

$91(28)$

- It depends on pathological features

$101(31)$

Indicate a priority order in the following statements related to GPs as follow-up providers

It depends mainly on cancer type:

- Priority level 1

- Priority level 2

- Priority level 3

- Priority level 4

- Priority level 5

$57(17)$

- Did not answer

Cancer therapies in progress or not:

- Priority level 1

- Priority level 2

- Priority level 3

- Priority level 4

- Priority level 5

67 (20)

- Did not answer

Patient's wish:

- Priority level 1

- Priority level 2

- Priority level 3

- Priority level 4

$76(23)$

- Priority level 5

At the fourth point of GPs' questionnaire, GPs were asked to provide an agree percentage to each of three statement (statement 1: the patient feels to be reassured by laboratory tests and imaging; statement 2: the patient is worried and had anxiety due to clinical visits and tests; statement 3: the patient, when close to diagnosis, is reassured from tests, after he becomes worried). The total sum had to be $100 \%$. The second point of medical oncologists' questionnaire aimed to figure out the best role of GPs in cancer patient care. Medical oncologists had to indicate a priority order in some tasks provided (promotion of prevention, risk individuation, follow-up management, therapies' side effects management, palliative care).

The table shows the percentage of oncologists providing a certain priority level for each task (from most important - number 1 - to least important - number 5). The fifth point of medical oncologists' questionnaire is related to GPs as follow-up providers. Medical oncologists were asked to order by relevance some factors that influence the possibility for a GP to become the main follow-up provider. These factors were: cancer type, cancer therapies in progress, patient's wish, year of diagnosis. The table shows the percentage of oncologists providing a certain level of relevance for each factor.

AIOM: Italian Association of Medical Oncology; GP: General practitioner; SIMG: Italian College of General Practitioner. 
Table 2. General practitioners', medical oncologists' and patients' perspectives (cont.).

Items

n (\%)

Medical oncologists' questionnaire (cont.)

Patient's wish (cont.):

- Did not answer

Year of diagnosis:

- Priority level 1

- Priority level 2

- Priority level 3

- Priority level 4

- Priority level 5

$26(8)$

- Did not answer $196(59)$

What do you think about GPs' commitment in informing patients about screening?

- It is adequate

- It is not adequate

$162(49)$

- Do not know

Are you used to write a patient's discharge letter for GPs?

- Yes

- No

$7(2)$

- Did not answer

What kind of strategy may improve relationship with GPs?

- Institution of common desks

- Conferences about follow-up and consensus conferences

- Formation programs

- Common guidelines

What do you think about the relationship between AIOM and SIMG?

- It's absent

- It's poor

- It's good

- It's excellent

- Did not answer

How much time do you spend to inform patients about relapse prevention?

- Always

- Enough

$120(36)$

- It's a priority

$70(22)$

- Very little

$6(2)$

How would you improve prevention promotion?

- Mass medias

$124(38)$

- Leaflets

- Websites and social networks

- Other

Patients' questionnaire

Which physician provides your follow-up?

- Medical oncologist

At the fourth point of GPs' questionnaire, GPs were asked to provide an agree percentage to each of three statement (statement 1: the patient feels to be reassured by laboratory tests and imaging; statement 2: the patient is worried and had anxiety due to clinical visits and tests; statement 3: the patient, when close to diagnosis, is reassured from tests, after he becomes worried). The total sum had to be $100 \%$. The second point of medical oncologists' questionnaire aimed to figure out the best role of GPs in cancer patient care. Medical oncologists had to indicate a priority order in some tasks provided (promotion of prevention, risk

individuation, follow-up management, therapies' side effects management, palliative care).

The table shows the percentage of oncologists providing a certain priority level for each task (from most important - number 1 - to least important - number 5). The fifth point of medical oncologists' questionnaire is related to GPs as follow-up providers. Medical oncologists were asked to order by relevance some factors that influence the possibility for a GP to become the main follow-up provider. These factors were: cancer type, cancer therapies in progress, patient's wish, year of diagnosis. The table shows the percentage of oncologists providing a certain level of relevance for each factor.

AIOM: Italian Association of Medical Oncology; GP: General practitioner; SIMG: Italian College of General Practitioner 
Table 2. General practitioners', medical oncologists' and patients' perspectives (cont.).

Patients' questionnaire (cont.)

Which physician provides your follow-up? (cont.)

$-\mathrm{GP}$

$90(26)$

- Several physicians (medical oncologist, surgeon, radiation oncologist...)

$30(9)$

- Do not answer

$24(6)$

Do you consult your GP for small symptoms related to cancer?

- Yes

- Sometimes

$112(32)$

- No

150 (43)

Is GP's opinion useful?

- Yes

60 (68)

- Sometimes

$22(25)$

- No $6(7)$

Do you trust your GP?

- Yes

- No

Do you consult your GP?

- Yes, regularly

102 (46)

- Sometimes

- Never

46 (21)

- Do not answer

(2)

If not, explain why:

- GPs lack of availability

60 (47)

- GPs lack of cancer expertise

60 (47)

- I had bad experiences in the past

$6(4)$

- Do not answer

(2)

Have you ever talked with your GP about cancer therapies' side effects?

- Yes

- Sometimes

- No 132 (38)

Do GPs provide information about clinical visits after treatment?

- Yes

- No

- Do not answer

In your opinion, collaboration between medical oncologists and GPs is:

- Useful

- Not useful

40 (11)

- Sometimes useful

$24(7)$

- Do not know

Nowadays, the above-mentioned collaboration is:

- Excellent

- Good

At the fourth point of GPs' questionnaire, GPs were asked to provide an agree percentage to each of three statement (statement 1: the patient feels to be reassured by laboratory tests and imaging; statement 2: the patient is worried and had anxiety due to clinical visits and tests; statement 3: the patient, when close to diagnosis, is reassured from tests, after he becomes worried). The total sum had to be $100 \%$. The second point of medical oncologists' questionnaire aimed to figure out the best role of GPs in cancer patient care. Medical oncologists had to indicate a priority order in some tasks provided (promotion of prevention, risk individuation, follow-up management, therapies' side effects management, palliative care).

The table shows the percentage of oncologists providing a certain priority level for each task (from most important - number 1 - to least important - number 5). The fifth point of medical oncologists' questionnaire is related to GPs as follow-up providers. Medical oncologists were asked to order by relevance some factors that influence the possibility for a GP to become the main follow-up provider. These factors were: cancer type, cancer therapies in progress, patient's wish, year of diagnosis. The table shows the percentage of oncologists providing a certain level of relevance for each factor.

AIOM: Italian Association of Medical Oncology; GP: General practitioner; SIMG: Italian College of General Practitioner. 
Table 2. General practitioners', medical oncologists' and patients' perspectives (cont.). Items n (\%)

Patients' questionnaire (cont.)

Nowadays, the above-mentioned collaboration is (cont.): 7 (14)

- Poor

- Absent

- Do not answer $45(18)$

How many tests are usually required by your GP in a clinical visit?

- One

$120(34)$

- Two

- Three

- More than three $96(28$

At the fourth point of GPs' questionnaire, GPs were asked to provide an agree percentage to each of three statement (statement

1: the patient feels to be reassured by laboratory tests and imaging; statement 2: the patient is worried and had anxiety due to clinical visits and tests; statement 3: the patient, when close to diagnosis, is reassured from tests, after he becomes worried). The total sum had to be $100 \%$. The second point of medical oncologists' questionnaire aimed to figure out the best role of GPs in cancer patient care. Medical oncologists had to indicate a priority order in some tasks provided (promotion of prevention, risk individuation, follow-up management, therapies' side effects management, palliative care).

The table shows the percentage of oncologists providing a certain priority level for each task (from most important - number 1 - to least important - number 5). The fifth point of medical oncologists' questionnaire is related to GPs as follow-up providers. Medical oncologists were asked to order by relevance some factors that influence the possibility for a GP to become the main follow-up provider. These factors were: cancer type, cancer therapies in progress, patient's wish, year of diagnosis. The table shows the percentage of oncologists providing a certain level of relevance for each factor.

AIOM: Italian Association of Medical Oncology; GP: General practitioner; SIMG: Italian College of General Practitioner.

\section{- GPs' perspectives}

GPs' questionnaire pointed out the proportion of patients which had a diagnosis of cancer: 230 GPs (61\%) declare to have more than 60 cancer patients. When asked which clinician takes charge of their follow-up, 286 (75\%) GPs declare that the follow-up management is provided by the collaboration between GPs and medical oncologists. With regard to followup investigation, 339 (89\%) GPs believe that patients undergo an excessive number of laboratory or instrumental tests, and only 25 (7\%) assume that testing is adequate. A question of the survey focused on follow-up strategy. After 2-3 years of disease free survival, 291 (76\%) GPs claim that follow-up should be provided by the collaboration between GPs and medical oncologists and that it should be personalized. Only 73 GPs (19\%) state that GP can take charge of patients' follow-up independently. When asked about their opinion about the value of collaboration with medical oncologists, more than a half ( $\mathrm{n}=177 ; 47 \%$ of GPs) considers it very important and 143 (38\%) GPs consider it quite important. The last point of the questionnaire investigated how much help could be provided by administration offices and a dedicated staff in GPs' follow-up management: 181 (48\%) GPs answer very much, 136 (36\%) GPs answer enough. Complete data are shown in Table 2.

\section{- Medical oncologists' perspectives}

Medical oncologists were asked about their relationship with GPs: 151 (46\%) report to have a poor relationship with GPs, 141 (43\%) to have a good relationship; for $23(7 \%)$ it is excellent, 14 (4\%) have no relationships. One hundred eighty-seven (57\%) oncologists deny the existence of a proper integration between local and hospital care. When asked how long after diagnosis GPs may replace medical oncologists in follow-up management, 101 (31\%) oncologists state that it depends on tumor characteristics, for $91(28 \%)$ of them it should depend on patient characteristics, for 87 (26\%) after 5 years. As regard to the oncologists' perception of GPs' commitment in informing patients about screening, 162 (49\%) oncologists believe it is not adequate. Communication between GPs and medical oncologists was also investigated. Three hundred twelve (95\%) medical oncologists assure to write a patient's discharge letter for GPs. One hundred thirty-nine (42\%) oncologists suggest establishment of common desks as a strategy to improve communication, 68 (21\%) conferences about follow-up and consensus conferences, a lower percentage suggest training programs and common guidelines. The last question was about prevention promotion: 128 (39\%) oncologists suggest leaflets as a strategy of prevention promotion, 124 (38\%) mass media, $48(15 \%)$ websites and social network (Table 2). 
Table 3. Significant associations $(p<0.05)$ between social-demographic features and answers provided by medical oncologists.

Relationship between GPs and medical oncologists

Absent, n (\%) Good, n (\%)

Excellent , $\mathrm{n} \quad$ Poor , $\mathrm{n}(\%)$

(\%)

Institution $(p=0.0003)$ :

- General hospital

- Research institute

9 (10.44)

$102(97.7)$

$17(16.41)$

$107(110)$

- Private clinic

$5(2.177)$

$13(20.3)$

0 (3.42)

$31(23)$

- Local service

$0(0.933)$

$12(8.73)$

1 (1.466)

$8(9.86)$

Age $(p=0.0110)$ :

- Younger than 40 years

$0(0.444)$

4 (4.15)

4 (0.698)

$2(4.69)$

- Older than 55 years

7 (4.9022)

$41(46.571)$

3 (7.7035)

$60(51.823)$

- Between 40 and 55 years

1 (4.0189)

47 (38.18)

$11(6.3155)$

32 (42.486)

Area $(p=0.0301)$ :

- Center

$6(5.0789)$

45 (48.249)

8 (7.9811)

56 (53.691)

- North

6 (3.1356)

25 (29.789)

4 (4.9274)

$36(33.148)$

2 (7.1546)

80 (67.968)

12 (11.243)

68 (75.634)

- South and Islands

6 (3.7098)

28 (35.243)

6 (5.8297)

44 (39.218)

Professional position $(p=0.0015)$ :

- Medical manager

- Full time

3 (2.959)

37 (28.11)

8 (4.6498)

19 (31.281)

- Fellow

4 (7.3312)

71 (69.647)

11 (11.521)

80 (77.502)

- Resident

3 (1.8107)

17 (17.202)

2 (2.8454)

19 (19.142)

Perception of a proper integration between hospital and local

4 (1.8991)

8 (18.041)

1 (2.9842)

30 (20.076)

care

Yes, n (\%)

No, n (\%)

Do not know, n (\%)

Institution $(p=0.0129)$ :

- General hospital

- Research institute

92 (90.27)

135 (136.52)

8 (8.2063)

- Private clinic

23 (18.822)

$26(28.467)$

0 (1.7111)

- Local service

3 (8.0667)

17 (12.2)

1 (0.7333)

Habit to write a discharge letter for the GP

3 (3.8413)

5 (5.8095)

$2(0.3492)$

Age $(p=0.0349)$ :

- Younger than 40 years

No, n (\%) Yes, n (\%)

- Older than 55 years

$10(5.6025) \quad 101(105.4)$

- Between 40 and 55 years

$1(4.5931) \quad 90(86.407)$

5 (5.8044)

110 (109.2)

Strategies suggested to improve prevention in the population Mass media, $\mathrm{n} \quad$ Leaflets, $\mathrm{n}(\%) \quad$ Internet, $\mathrm{n}(\%) \quad$ Do not know, $\mathrm{n}(\%)$ (\%)

Age $(p=0.0305)$ :

- Younger than 40 years

- Older than 55 years

50 (41.669)

27 (16.457)

1 (0.3502)

35 (34.161)

37 (35.596)

6 (13.492)

$0(0.2871)$

- Between 40 and 55 years

34 (43.17)

57 (44.984)

14 (17.05)

$0(0.3628)$

After how long GPs may replace medical oncologists as

follow-up providers

10 years, $\mathrm{n}(\%) \quad 5$ years, $\mathrm{n}(\%) \quad 3$ years, $\mathrm{n}(\%) \quad$ Never, $\mathrm{n}(\%)$

Area $(p=0.0006)$ :

- Center

8 (5.5994)

$24(19.038)$

3 (1.7918)

1 (3.3596)

- North

10 (12.776)

$46(43.438)$

5 (4.0883)

3 (7.6656)

- South and Islands

7 (6.6246)

15 (22.524)

0 (2.1199)

11 (3.9748)

According to pathological

features, $n$ (\%) patient, $\mathrm{n}(\%)$

Area $(p=0.0006)$ :

- Center

17 (21.726)

18 (19.486)

- North

58 (49.571)

$40(44.461)$

- South and Islands

22 (25.703)

$29(23.054)$

AIOM: Italian Association of Medical Oncology; GP: General practitioner; SIMG: Italian College of General Practitioner. 
RESEARCH ARTICLE Puglisi, Agostinetto, Gerratana et al.

\begin{tabular}{|c|c|c|c|}
\hline $\begin{array}{l}\text { Perception of GPs' commitment in informing patients about } \\
\text { screening }\end{array}$ & Yes, n (\%) & No, $n(\%)$ & Do not know, n (\%) \\
\hline \multicolumn{4}{|l|}{ Area $(p=0.0002)$} \\
\hline - Center & $3(6.9432)$ & $28(35.164)$ & $40(28.893)$ \\
\hline - North & $23(15.842)$ & $73(80.233)$ & $66(65.924)$ \\
\hline - South and Islands & $5(8.2145)$ & $56(41.603)$ & $23(34.183)$ \\
\hline Perception of the relationship between AIOM and SIMG & Absent, n (\%) & Good, n (\%) & Poor, $\mathrm{n}(\%)$ \\
\hline \multicolumn{4}{|l|}{ Professional position $(p=0.0208)$ : } \\
\hline - Medical manager & $7(4.2271)$ & $15(17.965)$ & $45(44.808)$ \\
\hline - Full time & $10(10.473)$ & $36(44.511)$ & $120(111.02)$ \\
\hline - Fellow & $3(2.5868)$ & $16(10.994)$ & $22(27.42)$ \\
\hline - Resident & $0(2.7129)$ & $18(11.53)$ & $25(28.757)$ \\
\hline
\end{tabular}

- Patients' perspectives

Patients were asked if they used to consult their GPs for small symptoms, defined as all adverse events that are perceived by patients as not being important enough to generate strong concerns that are not worth to be referred to the physicians. For such cancer related symptoms 150 (43\%) deny consulting their GPs, 88 (25\%) do it, and $112(32 \%)$ declare only sporadic consultations. When asked which physician provides their follow-up, 206 (59\%) indicate medical oncologists, 90 (26\%) several physicians (medical oncologist, surgeon, radiation oncologist) and only 30 (9\%) GPs. Two hundred twenty-two (63\%) patients trust their GPs, but 46 (21\%) never consult it (68 [31\%] do it sometimes and only 102 [46\%] do it regularly). The reasons why patients do not consult their GPs were examined: 60 (47\%) patients refer to GPs' lack of availability, 60 (47\%) lack of cancer expertise, six (4\%) had bad experiences in the past. One hundred thirty-four (38\%) patients have never talked with their GPs about cancer therapies' side effects. When asked if GPs provide information about clinical visits after treatment, $254(72 \%)$ patients deny it. On the other hand, collaboration between medical oncologists and GPs is perceived as useful by $256(73 \%)$ patients, even if, nowadays, the above mentioned collaboration is considered absent by $138(54 \%)$ patients (Table 2).

Table 3 reports significant associations observed by analyzing data collected from medical oncologists' questionnaire (threshold of statistical significance $\mathrm{p} \leq 0.05$ ). The relationship between oncologists and GPs is significantly associated with oncologist's age $(p=0.0110)$, type of institution $(p=0.0003)$, area of activity $(p=0.0301)$ and professional position $(\mathrm{p}=0.0015)$. The perception of a proper integration between hospital and local care is associated with the type of institution ( $p=0.0129)$. The habit to write a discharge letter for the GP and the strategies suggested to improve prevention in the population present a significant association with oncologist's age ( $\mathrm{p}=0.0349$ and 0.0305 , respectively). Answers to the question about after how long GPs may replace medical oncologists as follow-up providers are associated with the area of activity $(\mathrm{p}=0.0006)$. There is also a significant association between the perception of GPs' commitment in informing patients about screening and the area of activity ( $\mathrm{p}=0.0003)$ and between the perception of the relationship between AIOM and SIMG and the professional position $(\mathrm{p}=0.0208)$.

\section{Discussion}

The present study analyzed the results obtained thanks to a national survey that involved 380 GPs, 329 medical oncologists and 350 patients. More than $60 \%$ of GPs declared to have a high proportion of cancer patients. This suggests that surveillance is a daily practice issue that demands a tight multidisciplinary integration, in accordance with literature [28]. 75\% of GPs stated that follow-up should be provided by the collaboration between GPs and medical oncologists. Oncologists, on the other hand, point out that this collaboration is lacking (57\%). This view is supported also by the quality of relationship between medical oncologists and GPs, perceived as poor (according to $46 \%$ of oncologists) or absent (according to $4 \%$ of oncologists).

Personalization of follow-up is another hotspot [29]: the absolute majority of GPs (76\%) 
shares the idea that after $2-3$ years of disease-free survival, surveillance should be personalized and decided in collaboration with medical oncologists. Only $28 \%$ of oncologists share this point of view, stating that follow-up strategy should vary according to patients' characteristics. Among the remaining respondents, $26 \%$ state that GPs may replace oncologists after 5 years from diagnosis and $31 \%$ believe that follow-up strategy should depend on disease features.

However, the survey is consistent with the literature data on medical oncologists and GPs views about the value of a mutual collaboration [30]. Namely, $85 \%$ of GPs consider a shared patient care very important or quite important and $95 \%$ of medical oncologists are used to write a discharge letter for the GP. This relationship could be improved even further: common desks might enhance communication between GPs and medical oncologists.

Interestingly, our data differ from those presented by previous studies, especially when pointing out that patients usually prefer their own GPs to medical oncologists $[12,19-20,31]$. In these studies, although the reasons of choice are the same, up to $67 \%$ of patients prefer GPs to medical oncologists and more than half of them felt to be adequately followed by the referring physician. In our survey $43 \%$ of interviewed patients do not consult their GPs also for small symptoms related to cancer; $59 \%$ of patients' follow-up is provided by medical oncologists and only in $9 \%$ of cases by GPs. 37\% of patients do not trust their GPs and $21 \%$ never consult them. The reasons for such behavior include the lack of GPs' availability to support patients' needs, and a perceived poorer cancer expertise in respect to medical oncologists. The differences in choice among Italian patients and other countries patients are difficult to interpret due to the heterogeneity of survey methodology and could be only partially explained by local sociocultural and demographic characteristics as well as specific healthcare systems. One possible explanation, certainly speculative and not based on evidence, could be the different ease in accessing hospital of Italian patients compared with patients who live in America or Australia, thus conditioning the different preferences between GPs and oncologists.

The association analysis performed on the answers given by the medical oncologist offers some interesting insights. The type of institution where the oncologist works seems to be strictly connected with the quality of relationship between oncologists and GPs ( $p=0.0003)$. Medical oncologists based in a General Hospital tend to have a better relationship with GPs compared with those who work in a research institute (observed frequencies vs expected: 107, 31 and 110.41, 23.022, respectively). Similarly, medical oncologists based in a local service have a good relationship with GPs. This difference is probably due to closer contacts and similar workflows. Moreover, the type of institution is also associated with the oncologists' perception of the integration between local and hospital care $(p=0.0129)$. Medical oncologists who work in a private clinic deny the above-mentioned integration (observed frequency $=17$, expected frequency $=12.2$ ). Those who are based in a general hospital or in a research institute gave more positive feedbacks than expected.

Interestingly, the quality of relationship between medical oncologists and GPs seems to be influenced also by the oncologists' age $(p=0.0110)$. Younger oncologists (under 40 years) tend to be more negative especially compared with over 55-year-old colleagues. These data might reflect the heterogeneous age distribution among the GPs' subset. A total of $77 \%$ of GPs, at the time of the survey, were older than 55 years old. It is likely that older oncologists have a better relationship with GPs of the same age, rather than younger oncologists.

The oncologist's age seems to influence also the workflow organization. Older professionals tend to provide discharge letters more often $(\mathrm{p}=0.0349)$. This trend that is similar to quality in relationship with GPs. It suggests a key role of communication, in relationship quality.

The communication strategy differs also when it comes to patients' management. Medical oncologists aged less than 40 years focus mainly on mass media, web sites and social network, whereas their over 55-year-old colleagues suggest leaflets' distribution $(p=0.0305)$. The interpretation driven from these data reflects a different familiarity with technological devices by professionals of different age.

The area of activity seems to be also crucial in influencing GPs' relationship ( $\mathrm{p}=0.0301)$ and follow-up management. In the north of Italy, the relationship seems to be better and more integrated than in the south. Notably, a higher proportion of oncologists in the north state that GPs may replace medical oncologists as follow-up providers $(\mathrm{p}=0.0006)$. Among southern oncologists, $13 \%$ state that a GP can never 
replace a medical oncologist. Among northern oncologists, the majority state that it depends on pathological features (observed frequency $=58$, expected frequency $=49.571)$. The same interpretation can be provided on considering the association between area of activity and oncologists' perception of GPs' commitment in informing patients about screening $(\mathrm{p}=0.0002)$. Northern oncologists have a better opinion of GPs' commitment than southern colleagues do.

The last association found was between the quality of relationship between medical oncologists and GPs and oncologist's professional position ( $p=0.0015)$. Medical oncologists who work as medical managers or full-time consultants declare a better relationship with GPs rather than fellows and residents. Usually, professional positions as medical manager or full-time consultant are hold by older people than those who are fellows or residents: that could confirm the direction of the association between age and quality of relationship between oncologists and GPs.

Due to the exploratory intent of the survey, some results should be interpreted with caution. Most of the patients interviewed were very close to the time of diagnosis, a phase when the relationship with the oncologist is stronger, or even exclusive. Furthermore, GPs, medical oncologists and patients were invited to fill an online questionnaire. This method could select participants who share some characteristics as familiarity with technology or tendency to constantly update, leading to a potentially unbalanced population. Last, the answers provided were not verified by objective medical observations, as all participants referred to their own self-reported behavior.

In conclusion, follow-up is certainly an important challenge of modern oncology. Similarly to the phase focused on active treatment, it demands a multidisciplinary approach, but on the other hand its organization cannot rely on the common frameworks that characterize the workflow of different units of the same cancer center. Analyzing the feedback coming from all the actors that take part in this process, patients included, is precious in order to design common guidelines that are focused in optimizing both resources and efficiency in cancer survivorship. These feedback may vary according to different systems in different countries. Exploration of Italian setting might provide good practical targets for medical personnel to take, such as the need of a better communication among health providers (between GPs and medical oncologists, in particular) and the need of a more trustworthy relationship between patients and their GPs.

\section{Disclosure}

The paper is part of an Associazione Italiana di Oncologia Medica (AIOM) project on cancer survivorship.

Financial \& competing interests disclosure

F Puglisi had a consulting or advisory role for Celgene, Ipsen, Novartis, Pierre Fabre, Roche and Shionogi. G Numico had a consulting or advisory role for Lilly and Amgen. The authors would like to thank the Associazione Italiana di Oncologia Medica (AIOM) for having supported this study both logistically and financially. The authors have no other relevant affiliations or financial

\section{EXECUTIVE SUMMARY}

- There are a growing number of cancer survivors but a gold standard in surveillance management is currently lacking.

- We conducted a national survey: an online questionnaire was filled by 329 medical oncologists, 380 general practitioners (GPs) and 350 patients.

- The questionnaire included demographic information, adherence to guidelines, continuity and coordination of care, opinion on follow-up strategy and patients' satisfaction.

- Most GPs claim that follow-up should be provided by the collaboration between GPs and medical oncologists, after 2-3 years of disease-free survival.

- The majority of medical oncologists report to have a poor relationship with GPs.

- Patients tend to trust their GPs, but collaboration between medical oncologists and GPs is perceived as poor.

- According to answers provided, the collaboration between oncologists and GPs is considered poor and needs to be improved.

- Analyzing the feedbacks coming from all the actors that take part in this process could help in designing common guidelines that optimize both resources and efficiency. 
involvement with any organization or entity with a financial interest in or financial conflict with the subject matter or materials discussed in the manuscript apart from those disclosed.

No writing assistance was utilized in the production of this manuscript.

\section{Ethical conduct of research}

The authors state that they have obtained appropriate institutional review board approval or have followed the principles outlined in the Declaration of Helsinki for all human or animal experimental investigations. In addition, for investigations involving human subjects, informed consent has been obtained from the participants involved.

\section{Open access}

This work is licensed under the Creative Commons Attribution-NonCommercial 4.0 Unported License. To view a copy of this license, visit http://creativecommons. org/licenses/by-nc-nd/4.0/

\section{References}

Papers of special note have been highlighted as: - of interest

1 AIOM-AIRTUM. I numeri del cancro in Italia (2015).

www.aiom.it

2 Numico G, Pinto C, Gori S et al. Clinical and organizational issues in the management of surviving breast and colorectal cancer patients: attitudes and feelings of medical oncologists. PLoS ONE e101170 (2014).

- A survey aimed to assess attitudes and feelings of oncologists is considered preliminary to further initiatives toward more efficient models of survivorship care delivery.

3 Ofori S, Heddon MA, Griffis M. Toward a risk-based assessment of the adult cancer survivor: late effects of chemotherapy. Hosp. Pract. 37, 113-120 (2009) (1995).

4 McCabe MS, Partridge AH, Grunfeld E, Hudson MM. Risk-based healthcare, the cancer survivor, the oncologist, and the primary care physician. Semin. Oncol. 40, 804-812 (2013).

- Summarizes results of intervention studies implementing these elements in transitioning survivors from oncology to primary care providers for long-term follow-up care.

5 Siegel R, DeSantis C, Virgo K et al. Cancer treatment and survivorship statistics, 2012. CA Cancer J. Clin. 62, 220-241 (2012).

6 Rojas MP, Telaro E, Russo A et al. Follow-up strategies for women treated for early breast cancer. Cochrane Database Syst. Rev. (1) CD001768 (2005).

7 Grant M, Economou D. The evolving paradigm of adult cancer survivor care. Oncology Williston Park 22, 13-22, 27 (2008).

8 Ganz PA. Survivorship: adult cancer survivors. Prim. Care 36, 721-741 (2009).

9 Miller K, Mehta R, Abraham J, Opneja A, Jain RK. Patterns of Long-term cancer survivorship care in a National Cancer Institute-designated comprehensive cancer center. Am. J. Clin. Oncol. doi:10.1097/ COC.0000000000000217 (2015) (Epub ahead of print).

10 Johnson CE, Saunders CM, Phillips M et al. Randomized controlled trial of shared care for patients with cancer involving general practitioners and cancer specialists. J. Oncol. Pract. Am. Soc. Clin. Oncol. 11, 349-355 (2015).

11 Donnelly P, Hiller L, Bathers S, Bowden S, Coleman R. Questioning specialists' attitudes to breast cancer follow-up in primary care. Ann. Oncol. 18(9), 1467-1476 (2007).

12 Grunfeld E, Levine MN, Julian JA et al. Randomized trial of long-term follow-up for early-stage breast cancer: a comparison of family physician versus specialist care. J. Clin. Oncol. 24, 848-855 (2006).

- Tests the hypothesis that follow-up by the patient's family physician is a safe and acceptable alternative to specialist follow-up. This is important in order to define the best follow-up strategy in cancer patients.

13 O'Brien MA, Grunfeld E, Sussman J, Porter G, Mobilio MH. Views of family physicians about survivorship care plans to provide breast cancer follow-up care: exploration of results from a randomized controlled trial. Curr. Oncol. Tor. Ont. 22, 252-259 (2015).

14 Hahn EE, Ganz PA, Melisko ME et al. Provider perceptions and expectations of breast cancer posttreatment care: a University of California Athena Breast Health Network project. J. Cancer Surviv. Res. Pract. 7 , 323-330 (2013).

15 Puglisi F, Fontanella C, Numico G et al. Follow-up of patients with early breast cancer: is it time to rewrite the story? Crit. Rev. Oncol. Hematol. 91, 130-141 (2014).

- This review, illustrating the progress made in the treatment of metastatic disease and the rapid evolution of targeted therapy, considers the customization of the follow-up strategy according to molecular characteristics of the disease.

16 Grunfeld E, Hodgson DC, Del Giudice ME, Moineddin R. Population-based longitudinal study of follow-up care for breast cancer survivors. J. Oncol. Pract. Am. Soc. Clin. Oncol. 6, 174-181 (2010).

- Shows substantial variation in adherence to guidelines recommendations on follow-up care, for which effective intervention is possible.

17 Mayer EL, Gropper AB, Neville BA et al. Breast cancer survivors' perceptions of survivorship care options. J. Clin. Oncol. 30, 158-163 (2012).

18 Keating NL, Landrum MB, Guadagnoli E, Winer EP, Ayanian JZ. Surveillance testing among survivors of early-stage breast cancer. J. Clin. Oncol. 25, 1074-1081 (2007).

19 Bell RJ, Fradkin P, Robinson PJ, Schwarz M, Davis SR. Intended follow up of women with breast cancer at low risk of recurrence and at least 5 years from diagnosis. Intern. Med. J. 44, 332-338 (2014).

20 Brennan ME, Butow P, Marven M, Spillane AJ, Boyle FM. Survivorship care after breast cancer treatment - experiences and preferences of Australian women. Breast Edinb. Scotl. 20, 271-277 (2011).

21 Limon D, Perry S, Granot T, Gordon N, Stemmer N, Stemmer SM. ReCAP: perspectives of patients, caregivers, and medical staff on greetings in oncology practice: a prospective survey. J. Oncol. Pract. 12(2), 170-171; e188-e196 (2016)

22 Klemanski DL, Browning KK, Kue J. Survivorship care plan preferences of cancer survivors and healthcare providers: a systematic review and quality appraisal of the evidence. J. Cancer Surviv. 10(1), 71-86 (2016).

23 Potosky AL, Han PKJ, Rowland J et al. Differences between primary care physicians' and oncologists' knowledge, attitudes and practices regarding the care of cancer 
RESEARCH ARTICLE Puglisi, Agostinetto, Gerratana et al.

survivors. J. Gen. Intern. Med. 26(12), 1403-1410 (2011).

24 Mao JJ, Bowman MA, Stricker CT et al. Delivery of survivorship care by primary care physicians: the perspective of breast cancer patients. J. Clin. Oncol. 27(6), 933-938 (2009).

25 Wind J, Duineveld LA, van der Heijden RP, van Asselt KM, Bemelman WA, van Weert HC. Follow-up after colon cancer treatment in The Netherlands; a survey of patients, GPs, and colorectal surgeons. Eur. J. Surg. Oncol. 39(8), 837-843 (2013).

26 Cheung WY, Aziz N, Noone A-M et al. Physician preferences and attitudes regarding different models of cancer survivorship care: a comparison of primary care providers and oncologists. J. Cancer Surviv. Res. Pract. 7(3), 343-354 (2013).

27 Del Giudice ME, Grunfeld E, Harvey BJ, Piliotis E, Verma S. Primary care physicians' views of routine follow-up care of cancer survivors. J. Clin. Oncol. 27(20), 3338-3345 (2009).

28 Grant M, Economou D, Ferrell B, Uman G. Educating healthcare professionals to provide institutional changes in cancer survivorship care. J. Cancer Educ. 27, 226-232 (2012).

29 McCabe MS, Bhatia S, Oeffinger KC et al. American Society of Clinical Oncology statement: achieving high-quality cancer survivorship care. J. Clin. Oncol. 31, 631-640 (2013).

- Describes the key initiatives individuated by the American Society of Clinical Oncology to assist the oncology community in the delivery of quality survivorship care.

30 Grunfeld E, Earle CC. The interface between primary and oncology specialty care: treatment through survivorship. J. Natl Cancer Inst. Monogr. 2010, 25-30 (2010).

31 Ganz PA, Kwan L, Stanton AL et al. Quality of life at the end of primary treatment of breast cancer: first results from the moving beyond cancer randomized trial. J. Natl Cancer Inst. 96, 376-387 (2004). 